\title{
CONJUGACY CORRESPONDENCES: A UNIFIED VIEW
}

\author{
BY
}

\author{
L. MCLINDEN(1)
}

ABSTRACT. As preparation for a duality theory for saddle programs, a partial conjugacy correspondence is developed among equivalence classes of saddle functions. Three known conjugacy correspondences, including Fenchel's correspondence among convex functions and Rockafellar's extension of it to equivalence classes of saddle functions, are shown to be degenerate special cases. Additionally, two new correspondences are brought to light as further special cases. Various questions are answered concerning the lower and upper closures and effective domain of the resulting equivalence class, as well as the effect the correspondence has on the related purely convex function and the subdifferential mapping.

Introduction. Conjugacy correspondences are the fundamental vehicle for applying the powerful concept of duality to extremum problems involving convex functions or saddle functions. The most basic and probably most familiar of these correspondences, and the one from which the others derive, is Fenchel's conjugacy correspondence among convex functions [2], [3]. Other important conjugacy correspondences are Rockafellar's extension of Fenchel's correspondence to (equivalence classes of ) saddle functions [8] and the "partial" conjugacy correspondence relating convex functions to (equivalence classes of ) saddle functions (Rockafellar [9]; see also Moreau [6]). A thorough treatment of these three conjugacy correspondences in finite-dimensional spaces, together with some of their applications to duality in convex extremum problems, can be found in [10]. In the infinite-dimensional case, the convex conjugacy correspondence is developed in Brondsted [1] and Moreau [7], while the partial conjugacy and saddle conjugacy correspondences are presented in Rockafellar [11]. The literature contains many related papers by these and other researchers too numerous to mention here.

In this paper we develop in finite-dimensional spaces a new conjugacy correspondence which includes the three mentioned above as degenerate special cases.

Received by the editors November 7, 1972 and, in revised form, January 16, 1973.

AMS (MOS) subject classifications (1970). Primary 90C25, 90C30, 90C99, 90D05; Secondary 26A51, 49A40, 49B40.

Key words and phrases. Convex analysis, conjugacy correspondences, minimax theory, saddle programs, duality, conjugate saddle functions, equivalence classes, closure formulas, subdifferential formulas.

(1) Sponsored by the United States Army under Contract DA-31-124-ARO-D-462 at the Mathematics Research Center, University of Wisconsin-Madison.

Copyright $\odot 1975$, American Mathematical Society 
It is a one-to-one symmetric correspondence among (equivalence classes of) saddle functions. It is basically an extension of Rockafellar's saddle conjugacy correspondence to cover the cases in which not all of the arguments are operated upon. For a saddle function resulting from this new "partial" conjugacy operation, we answer basic questions about its subdifferential, effective domain, lower and upper closure, and the related purely convex function. The proofs involve applying recent results concerning two dual ways of combining a saddle function with a linear transformation [4], and as preliminary lemmas we establish two new results concerning these dual operations.

The principal goal of this paper is actually to provide some of the tools needed for a perturbational duality theory for saddle programs paralleling Rockafellar's for convex programs [10]. This theory is already available in preliminary form [5]. By taking various coordinate spaces to be zero-dimensional, it includes Rockafellar's theory as a degenerate special case. A subsidiary goal here, however, is to present a unifying framework which encompasses the three important conjugacy correspondences mentioned above. This framework is provided by the new partial conjugacy correspondence for saddle functions, again using the device of taking various combinations of coordinate spaces to be zero-dimensional. In addition to the three known correspondences, the framework also gives as degenerate special cases two new conjugacy correspondences among saddle functions.

Results and discussion. The partial conjugacy correspondence developed here deals with all the closed proper saddle functions defined on some fixed product space $R^{\mu} \times R^{\nu}$. The correspondence depends not only on the product representation $R^{\mu} \times R^{\nu}$ but also on a further representation of each of $R^{\mu}$ and $R^{\nu}$ as the product of two spaces. Throughout, we consider as fixed the representations

where

$$
R^{\mu}=U \times X, \quad R^{\nu}=V \times Y,
$$

$$
U=R^{p}, \quad V=R^{q}, \quad X=R^{m}, \quad Y=R^{n},
$$

and $p, m, q, n$ are nonnegative integers satisfying

$$
\mu=p+m, \quad \nu=q+n .
$$

Thus, we allow any of the factor spaces to be the degenerate zero-dimensional topological vector space over the reals. It will be helpful to use the notation

that is,

$$
U^{\prime}=Z, \quad V^{\prime}=W, \quad X^{\prime}=S, \quad Y^{\prime}=T,
$$

$$
Z=R^{p}, W=R^{q}, \quad S=R^{m}, \quad T=R^{n} .
$$

The central result of this paper is the following. (The definitions used, unless otherwise stated, are those in Rockafellar [10].) 
THEOREM 1. Let $M$ be a closed proper saddle function on $(U \times X) \times$ $(V \times Y)$. For definiteness, suppose $M$ is concave-convex. Then the functions $H_{1}$ and $H_{2}$ defined by

and

$$
H_{1}(z, y, w, x)=\sup _{v} \inf _{u}\{\langle u, z\rangle+\langle v, w\rangle-M(u, x, v, y)\}
$$

$$
H_{2}(z, y, w, x)=\inf _{u} \sup _{v}\{\langle u, z\rangle+\langle v, w\rangle-M(u, x, v, y)\}
$$

belong to an equivalence class $[H]$ of closed proper concave-convex functions on $(Z \times Y) \times(W \times X)$, where $[H]$ depends only on the equivalence class $[M]$ containing M. Moreover, for any element $H$ of $[H]$, the functions $M_{1}$ and $M_{2}$ defined by

and

$$
M_{1}(u, x, v, y)=\sup _{w} \inf _{z}\{\langle z, u\rangle+\langle w, v\rangle-H(z, y, w, x)\}
$$

$$
M_{2}(u, x, v, y)=\inf _{z} \sup _{w}\{\langle z, u\rangle+\langle w, v\rangle-H(z, y, w, x)\}
$$

belong to $[M]$.

Note trivially that if $U$ (resp. $V$ ) is zero-dimensional, the expressions "inf $u$ ", "inf $z$ " and " $\langle u, z\rangle$ " (resp. "sup $v "$, "sup ${ }_{w}$ " and " $\langle v, w\rangle$ ") can be omitted from the formulas in the theorem.

Theorem 1 sets up a one-to-one symmetric correspondence between the equivalence classes of closed proper concave-convex functions defined on $(U \times X) \times(V \times Y)$ and those defined on $(Z \times Y) \times(W \times X)$. We call it the partial conjugacy correspondence for saddle functions. The operation of passing from $[M]$ to $[H]$ is called taking the partial conjugate of $[M]$ in $U$ and $V$, and $[H]$ itself is called the partial conjugate of $[M]$ in $U$ and $V$. Note that, given a closed proper concave-convex function $M$ on $R^{\mu} \times R^{\nu}$, there are many different such partial conjugacy correspondences which can be applied to $[M]$, one for each of the different ways of representing $R^{\mu}$ and $R^{\nu}$ as $R^{\mu}=$ $U \times X$ and $R^{\nu}=V \times Y$.

For the remainder of the paper we consider $[M]$ and $[H]$ fixed as in Theorem 1.

Given the new correspondence established by Theorem 1, a number of questions naturally arise concerning the nature of, and relationship between, various objects associated with $[H]$ and $[M]$. For example: What are the least and greatest elements of $[H]$ ? What is the relationship of the subdifferential of $[H]$ to that of $[M]$, and similarly for effective domains and related convex functions? These are questions which must be answered if one is to make extensive use of the correspondence. Before dealing with them, though, we shall first give examples describing how the three conjugacy correspondences mentioned in the Introduction are included in this one. 
An observation needed for the first example is that closed proper convex (or concave) functions can be viewed as closed proper saddle functions which are degenerate in one of the two arguments, and vice versa. Indeed, suppose that $f$ is any closed proper convex function on $V$. Then $V$ can be identified with $X \times V$, where $X$ is the degenerate zero-dimensional space, and $f$ can be identified with the function $K$ on $X \times V$ given by $K(x, v)=f(v)$. The fact that $f$ is closed proper convex with effective domain $\operatorname{dom} f$ implies easily that $K$ is closed proper concave-convex with effective domain $X \times \operatorname{dom} f$. This procedure can also be reversed, starting with a closed proper concave-convex function $K$ on $X \times V$, where $X$ is zero-dimensional. Notice that such a saddle function $K$ is both concave-closed and convex-closed, and hence is necessarily the unique element of its equivalence class $[K]$. By abusing notation somewhat, we can thus write $[K]=f$ to describe the above situation.

EXAMPLE 1 (Fenchel's conjugacy correspondence for convex functions). Suppose $U, X$ and $Y$ are all zero-dimensional and that $M$ is given by

$$
M(u, x, v, y)=f(v),
$$

where $f$ is a closed proper convex function on $V$. Then for $i=1$ and 2,

and

$$
H_{i}(z, y, w, x)=\sup _{v}\{\langle v, w\rangle-f(v)\}=f^{*}(w)
$$

$$
M_{i}(u, x, v, y)=\sup _{w}\left\{\langle w, v\rangle-f^{*}(w)\right\}=f(v) .
$$

In other words, the correspondence $[M] \leftrightarrow[H]$ reduces to $f \leftrightarrow f^{*}$.

For the remaining examples we need an elaboration of the observation above. Namely, in a saddle function (and hence, by the previous observation, in a concave or convex function) one can add or delete "trivial" arguments without affecting the properties of being closed and proper, where a "trivial" argument is one ranging over a zero-dimensional space. To illustrate, consider a closed proper concave-convex function $L \cdot$ on $(U \times X) \times Y$, where $U$ is zero-dimensional. Then the function $K$ on $X \times Y$ given by $K(x, y)=L(u, x, y)$ is closed proper concave-convex on $X \times Y$. Clearly this procedure can also be reversed in order to add a trivial argument. By abusing notation again, we can describe this situation by writing $[L]=[K]$.

In the next example and subsequently, we use the lower and upper bar notation to denote the least and greatest elements of an equivalence class of saddle functions. Thus, if $K$ is a saddle function, then $\underline{K}$ is the least, and $\bar{K}$ the greatest, element of $[K]$.

EXAMPLE 2 (Rockafellar's conjugacy correspondence for saddle functions). Suppose $X$ and $Y$ are zero-dimensional and that $M$ is given by

$$
M(u, x, v, y)=K(u, v) \text {, }
$$


where $K$ is a closed proper concave-convex function on $U \times V$. Then and

$$
\begin{aligned}
& H_{1}(z, y, w, x)=\sup _{v} \inf _{u}\{\langle u, z\rangle+\langle v, w\rangle-K(u, v)\}=\underline{K}^{*}(z, w), \\
& H_{2}(z, y, w, x)=\inf _{u} \sup _{v}\{\langle u, z\rangle+\langle v, w\rangle-K(u, v)\}=\bar{K}^{*}(z, w),
\end{aligned}
$$

$$
\begin{aligned}
& M_{1}(u, x, v, y)=\sup _{w} \inf _{z}\left\{\langle z, u\rangle+\langle w, v\rangle-K^{*}(z, w)\right\}=\underline{K}(u, v), \\
& M_{2}(u, x, v, y)=\inf _{z} \sup _{w}\left\{\langle z, u\rangle+\langle w, v\rangle-K^{*}(z, w)\right\}=\bar{K}(u, v) .
\end{aligned}
$$

(To justify the use of $\underline{K}$ and $\bar{K}$ in the last two formulas, we have made use of Theorem 2 below, which identifies the least and greatest elements of an equivalence class obtained by partial conjugacy.) In other words, the correspondence $[M] \leftrightarrow[H]$ reduces to $[K] \leftrightarrow\left[K^{*}\right]$.

For the next example we introduce some terminology. According to Theorem 34.2 of [10] (or Theorem 4 of [11]), the closed proper convex functions $f$ on $X \times W$ and the equivalence classes $[K]$ of closed proper concave-convex functions on $X \times V$ are in one-to-one correspondence via the "partial conjugacy" formulas

$$
f(x, w)=\sup _{v}\{\langle v, w\rangle-K(x, v)\} \text { and } K(x, v)=\sup _{w}\{\langle w, v\rangle-f(x, w)\} .
$$

We shall call the $f$ related to $[K]$ in this manner the convex parent of $[K]$ and denote it by $f_{K}$. A similar one-to-one correspondence is established between the equivalence classes $[K]$ as above and the closed proper concave functions $g$ on $S \times V$ by means of the formulas

$$
\left.g(s, v)=\inf _{x}\{\langle x, s\rangle-K(x, v)\} \text { and } \bar{K}(x, v)=\inf _{s} i\langle s, x\rangle-g(s, v)\right\} .
$$

We shall call the $g$ related to $[K]$ in this manner the concave parent of $[K]$ and denote it by $g_{K}$. Note that this usage of the terms "convex parent" and "concave parent" differs by some minus signs from the usage in Rockafellar [9]. The connection between the parents just defined and the bifunction terminology and $\Omega(\cdot)$ notation employed in Theorem 34.2 of [10] is simply described: $f_{K}$ satisfies the relation $f_{K}(x, w)=(F x)(w)$, where $F$ is the convex bifunction from $X$ to $W$ corresponding to $[K]$ by means of the formula $\Omega(F)=[K]$, while $g_{K}$ satisfies the relation $g_{K}(s, v)=\left(F^{*} v\right)(s)$, where $F^{*}$ is the concave bifunction from $V$ to $S$ (the adjoint of $F$ ) corresponding to $[K]$ by means of the formula $\Omega\left(F^{*}\right)=[K]$. The next example shows how the partial conjugacy correspondences just described are included in the new one.

EXAMPLE 3 (Correspondence between saddle functions and purely convex or purely concave functions). Suppose first that $U$ and $Y$ are zero- 
dimensional and that $M$ is given by $M(u, x, v, y)=K(x, v)$, where $K$ is a closed proper concave-convex function on $X \times V$. Then for $i=1$ and 2,

$$
H_{i}(z, y, w, x)=\sup _{v}\{\langle v, w\rangle-K(x, v)\}=f_{K}(x, w)
$$

and

$$
M_{i}(u, x, v, y)=\sup _{w}\left\{\langle w, v\rangle-f_{K}(x, w)\right\}=\underline{K}(x, v) .
$$

(The use of $\underline{K}$ here follows by Theorem 2 below.) Thus, $[M] \leftrightarrow[H]$ reduces to $[K] \leftrightarrow f_{K}$. On the other hand, suppose that $X$ and $V$ are zerodimensional and $M$ is given by $M(u, x, v, y)=L(u, y)$, where $L$ is a closed proper concave-convex function on $U \times Y$. Then for $i=1$ and 2 ,

and

$$
H_{i}(z, y, w, x)=\inf _{u}\{\langle u, z\rangle-L(u, y)\}=g_{L}(z, y)
$$

$$
M_{i}(u, x, v, y)=\inf _{z}\left\{\langle z, u\rangle-g_{L}(z, y)\right\}=\bar{L}(u, y) .
$$

(The element $\bar{L}$ is justified by Theorem 2.) Thus, $[M] \leftrightarrow[\dot{H}]$ reduces to $[L] \leftrightarrow g_{L}$.

An examination of the various other possible ways of picking some of the coordinate spaces $U, X, V$ and $Y$ to be zero-dimensional reveals basically two new (nontrivial) conjugacy correspondences. Both relate saddle functions to saddle functions.

EXAMPLE 4. Suppose $U$ is zero-dimensional and $M$ is given by $M(u, x, v, y)=P(x, v, y)$, where $P$ is closed proper concave-convex on $X \times$ $(V \times Y)$. Then the equivalence class $[H]$ is of the form $H(z, y, w, x)=$ $Q(y, w, x)$, where $Q$ is closed proper concave-convex on $Y \times(W \times X)$. For each element $\widetilde{P}$ of $[P]$ the function

$$
(y, w, x) \rightarrow \sup _{v}\{\langle v, w\rangle-\widetilde{P}(x, v, y)\}
$$

belongs to $[Q]$, while for each element $\widetilde{Q}$ of $[Q]$ the function

$$
(x, v, y) \rightarrow \sup _{w}\{\langle w, v\rangle-\widetilde{Q}(y, w, x)\}
$$

belongs to $[P]$. From Theorem 2 it follows, moreover, that

and

$$
Q(y, w, x)=\sup _{v}\{\langle v, w\rangle-\bar{P}(x, v, y)\}
$$

$$
\underline{P}(x, v, y)=\sup _{w}\{\langle w, v\rangle-\bar{Q}(y, w, x)\} \text {. }
$$

Example 5. Suppose $Y$ is zero-dimensional and $M$ is given by $M(u, x, v, y)=P(u, x, v)$, where $P$ is closed proper concave-convex on $(U \times X) \times V$. Then the equivalence class $[H]$ is of the form $H(z, y, w, x)=$ $Q(z, w, x)$, where $Q$ is closed proper concave-convex on $Z \times(W \times X)$. For each element $\widetilde{P}$ of $[P]$ the functions 


$$
\begin{aligned}
& Q_{1}(z, w, x)=\sup _{v} \inf _{u}\{\langle u, z\rangle+\langle v, w\rangle-\widetilde{P}(u, x, v)\}, \\
& Q_{2}(z, w, x)=\inf _{u} \sup _{v}\{\langle u, z\rangle+\langle v, w\rangle-\widetilde{P}(u, x, v)\}
\end{aligned}
$$

belong to $[Q]$, while for each $\tilde{Q} \in[Q]$ the functions

$$
\begin{aligned}
& P_{1}(u, x, v)=\sup _{w} \inf _{z}\{\langle z, u\rangle+\langle w, v\rangle-\widetilde{Q}(z, w, x)\}, \\
& P_{2}(u, x, v)=\inf _{z} \sup _{w}\{\langle z, u\rangle+\langle w, v\rangle-\widetilde{Q}(z, w, x)\}
\end{aligned}
$$

belong to $[P]$. It follows from Theorem 2 that actually $Q_{2}=\bar{Q}$ and $P_{1}=$ $\underline{P}$.

We turn now to the questions mentioned following Theorem 1. These are answered by the next four theorems. From them the reader can easily deduce as corollaries assertions about the five "degenerate" conjugacy correspondences given in Examples 1-5. In the case of the three known correspondences (Examples 1-3), the resulting corollaries are known theorems.

The first of these theorems identifies the "extreme" elements of an equivalence class resulting from the partial conjugacy operation. For convenience, in the formulas we employ some new notation. Namely, if $f(p, x)$ is any function which is convex or concave in $x$ for each fixed $p$ (thought of as a parameter), then for each $p$ the closure (in the sense of convex function theory) of $f(p, \cdot)$ evaluated at $x$ is denoted by $\mathrm{cl}_{x} f(p, x)$.

THEOREM 2. The least element $\underline{H}$ and greatest element $\bar{H}$ of $[H]$ are given by

$$
\underline{H}(z, y, w, x)=\sup _{v} \mathrm{cl}_{x} \inf _{u}\{\langle u, z\rangle+\langle v, w\rangle-\widetilde{M}(u, x, v, y)\}
$$

and

$$
\left.\bar{H}(z, y, w, x)=\inf _{u} \mathrm{cl}_{y} \sup _{v}\{u, z\rangle+\langle v, w\rangle-\tilde{M}(u, x, v, y)\right\},
$$

where $\tilde{M}$ is any element of $[M]$.

Trivially, if $X$ (resp. $Y$ ) is zero-dimensional the closure operation " $\mathrm{cl}_{x}$ " (resp. "cly") can be omitted from the formula for $\underline{H}$ (resp. $\bar{H}$ ). These closure operations cannot be omitted in the general case, however. (For example, consider $[M]$ of the form $M(u, x, v, y)=K(x, v)$, where $U$ is not zero-dimensional and $K$ is closed proper concave-convex with $\operatorname{dom}_{1} K \neq X$.)

The next theorem characterizes the subdifferential of $[H]$. Roughly speaking, $\partial H$ is the "partial inverse" of $\partial M$. 
THEOREM 3. The condition $(z, s, w, t) \in \partial M(u, x, v, y)$ is equivalent to the condition $(u,-t, v,-s) \in \partial H(z, y, w, x)$. Moreover, these conditions imply for any element $\widetilde{M}$ of $[M]$ and any element $\widetilde{H}$ of $[H]$ that

(i) $(u, v)$ is a saddle point of $\langle\cdot, z\rangle+\langle\cdot, w\rangle-\widetilde{M}(\cdot, x, \cdot, y)$;

(ii) $(z, w)$ is a saddle point of $\langle\cdot, u\rangle+\langle\cdot, v\rangle-\widetilde{H}(\cdot, y, \cdot, x)$; and

(iii) $\widetilde{M}(u, x, v, y)+\widetilde{H}(z, y, w, x)=\langle u, z\rangle+\langle v, w\rangle$.

Condition (i) of Theorem 3 implies that the extrema appearing in the definitions of $H_{1}$ and $H_{2}$ are attained by $(u, v)$. In fact, it is not hard to show (cf. Theorem 6 of [4]) that the pair $(u, v)$ actually yields attainment of the extrema involved in $\underline{H}$ and $\bar{H}$ in a certain natural sense. Of course, similar remarks apply to condition (ii) and the functions $M_{1}, M_{2}, \underline{M}$ and $\bar{M}$.

It follows immediately from Theorem 3 that the projection of dom $\partial H$ onto $Y \times X$ is the same (modulo the isometry $(y, x) \rightarrow(x, y)$ ) as the projection of dom $\partial M$ onto $X \times Y$. The next theorem says that the same result holds for effective domains.

THEOREM 4. The projections of $\operatorname{dom}_{1} M$ and $\operatorname{dom}_{2} H$ onto $X$ are equal, and the projections of $\operatorname{dom}_{2} M$ and $\operatorname{dom}_{1} H$ onto $Y$ are equal.

Finally, the effect of partial conjugacy on concave and convex parents can be simply described.

THEOREM 5. The convex and concave parents of $[M]$ and $[H]$ satisfy the identities

$$
f_{H}(z, y, v, s)=-g_{M}(z,-s, v, y)
$$

and

$$
g_{H}(u, t, w, x)=-f_{M}(u, x, w,-t) .
$$

This theorem says basically that the convex (resp. concave) parent of $[H]$ is the same (modulo minus signs and reordering variables) as the concave (resp. convex) parent of $[M]$.

A similar assertion can be established relating the ordinary conjugate $\left[H^{*}\right]$ of $[H]$ to $[M]$. It is that the (ordinary) conjugate of $[H]$ is the same (modulo minus signs and reordering variables) as the partial conjugate of $[M]$ in $X$ and $Y$. The implications of this are important for developing a duality theory for saddle programs [5].

Proofs. In the proofs of Theorems 1-5, extensive use will be made of those theorems of [4] which concern two dual ways of combining a saddle function with a linear transformation. But before we can apply those results, we need to dualize the hypothesis on which they depend, thereby converting it into a form 
we can verify. For this it is convenient to introduce the notions of "recession function" and "recession cone" for a saddle function.

First, recall the definitions of these terms as used in convex function theory. The recession function of a proper convex function $f$ is the function

$$
(\operatorname{rec} f)(y)=\sup \left\{f\left(y^{\prime}+y\right)-f\left(y^{\prime}\right) \mid y^{\prime} \in \operatorname{dom} f\right\},
$$

while the recession cone of $f$ is the set

$$
\text { rec cone } f=\{y \mid(\operatorname{rec} f)(y) \leqslant 0\} \text {. }
$$

Similarly, the recession function of a concave function $g$ is the function

$$
(\operatorname{rec} g)(x)=\inf \left\{g\left(x^{\prime}+x\right)-g\left(x^{\prime}\right) \mid x^{\prime} \in \operatorname{dom} g\right\} \text {, }
$$

and the recession cone of $g$ is the set

$$
\text { rec cone } g=\{x \mid(\operatorname{rec} g)(x) \geqslant 0\} \text {. }
$$

(This notation differs from that in [10].)

Now suppose $K$ is a saddle function on $R^{m} \times R^{n}$, where for definiteness we suppose $K$ is concave-convex. We define the convex recession function of $K$ to be the function

$$
\left(\operatorname{rec}_{2} K\right)(y)=\sup \left\{(\operatorname{rec} K(x, \cdot))(y) \mid x \in \operatorname{ri}\left(\operatorname{dom}_{1} K\right)\right\}
$$

and the convex recession cone of $K$ to be the set

$$
\text { rec cone }{ }_{2} K=\left\{y \mid\left(\operatorname{rec}_{2} K\right)(y) \leqslant 0\right\} \text {. }
$$

Similarly, we define the concave recession function of $K$ to be the function

$$
\left(\operatorname{rec}_{1} K\right)(x)=\inf \left\{(\operatorname{rec} K(\cdot, y))(x) \mid y \in \operatorname{ri}\left(\operatorname{dom}_{2} K\right)\right\}
$$

and the concave recession cone of $K$ to be the set

$$
\text { rec cone }{ }_{1} K=\left\{x \mid\left(\operatorname{rec}_{1} K\right)(x) \geqslant 0\right\} \text {. }
$$

The main importance of the (concave and convex) recession functions of $K$ rests on the following fact.

Lemma 1 (Rockafellar [10, Theorem 37.2). Assume $K$ is a closed proper concave-convex function on $R^{m} \times R^{n}$. Then

$$
\operatorname{rec}_{1} K=\inf \left\{\langle\cdot, s\rangle \mid s \in \operatorname{dom}_{1} K^{*}\right\}
$$

and

$$
\operatorname{rec}_{2} K=\sup \left\{\langle\bullet, t\rangle \mid t \in \operatorname{dom}_{2} K^{*}\right\} .
$$

From the lemma and Theorem 13.2 of [10] it follows that, when $K$ is closed and proper, the convex (resp. concave) recession function of $K$ is a positively homogeneous closed proper convex (resp. concave) function. Furthermore, since dom $K^{*}$ depends only on $[K]$, the lemma also shows that the recession functions (and hence the recession cones) of $K$ depend only on $[K]$. 
We are now ready to dualize the hypothesis involved in Theorems 4,5 and 6 of [4]. This is done in the following easy result.

Lemma 2. Assume $K$ is a closed proper concave-convex function on $R^{m} \times R^{n}$, and let $A=A_{1} \times A_{2}$ be a product linear transformation from $R^{m} \times R^{n}$ to $R^{p} \times R^{q}$. Then for $j=1$ and 2 ,

$$
\text { (range } \left.A_{j}^{*}\right) \cap \operatorname{ri}\left(\operatorname{dom}_{j} K^{*}\right) \neq \varnothing
$$

occurs if and only if $A_{j}^{-1}\{0\} \cap\left(\mathrm{rec}\right.$ cone $\left.{ }_{j} K\right)$ is a subspace. (Here $A_{j}^{*}$ is the adjoint of $A_{j}$.)

Proof. We prove the assertion only for $j=2$, as the proof for $j=1$ is analogous. Put $L=$ range $A_{2}^{*}$ and $D^{*}=\operatorname{dom}_{2} K^{*}$. Then we have to show that $L \cap$ i $D^{*} \neq \varnothing$ if and only if $L^{\perp} \cap\left(\right.$ rec cone $\left.{ }_{2} K\right)$ is a subspace. But $L \cap$ ri $D^{*}=\varnothing$ if and only if there exists a hyperplane separating $L$ and $D^{*}$ properly, that is, if and only if there exists a $y$ such that

$$
\inf \{\langle y, t\rangle \mid t \in L\} \geqslant \sup \left\{\langle y, t\rangle \mid t \in D^{*}\right\}
$$

and

$$
\sup \{\langle y, t\rangle \mid t \in L\}>\inf \left\{\langle y, t\rangle \mid t \in D^{*}\right\} .
$$

Now $\sup \left\{\langle y, t\rangle \mid t \in D^{*}\right\}=\left(\operatorname{rec}_{2} K\right)(y)$ and inf $\left\{\langle y, t\rangle \mid t \in D^{*}\right\}=-\left(\operatorname{rec}_{2} K\right)(-y)$ by Lemma 1 , and since $L$ is a subspace,

$$
\inf \{\langle y, t\rangle \mid t \in L\}=\left\{\begin{array}{lll}
0 & \text { if } & y \in L^{\perp}, \\
-\infty & \text { if } & y \notin L^{\perp},
\end{array}\right.
$$

and

$$
\sup \{\langle y, t\rangle \mid t \in L\}=\left\{\begin{array}{ccc}
0 & \text { if } & y \in L^{\perp}, \\
+\infty & \text { if } & y \notin L^{\perp}
\end{array}\right.
$$

Thus, $L \cap$ ri $D^{*}=\varnothing$ if and only if there exists a $y \in L^{\perp}$ such that $\left(\operatorname{rec}_{2} K\right)(y) \leqslant$ 0 and $\left(\operatorname{rec}_{2} K\right)(-y)>0$. Taking contrapositives, we obtain that $L \cap$ ri $D^{*} \neq$ $\varnothing$ occurs if and only if $L^{\perp} \cap\left(\right.$ rec cone $_{2} K$ ) is closed under scalar multiplication by -1 . But since $L^{\perp}$ and rec cone ${ }_{2} K$ are convex cones containing the origin, this concludes the proof.

Proof of Theorem 1. Define a product linear transformation $A=$ $A_{1} \times A_{2}$ and a saddle function $K$ by

$$
A_{1}(z, v, y)=(z, y), \quad A_{2}(w, u, x)=(w, x),
$$

and

$$
K(z, v, y, w, u, x)=\langle u, z\rangle+\langle v, w\rangle-M(u, x, v, y)
$$


Clearly $K$ is closed proper concave-convex on $(Z \times V \times Y) \times(W \times U \times X)$. The first half of the theorem will follow from Theorem 5 of [4], provided we can show that (range $\left.A^{*}\right) \cap$ ri $\left(\operatorname{dom} K^{*}\right) \neq \varnothing$. But for this, in view of Lemma 2 it suffices to show that $A_{j}^{-1}\{0\} \cap\left(\right.$ rec cone $\left.{ }_{j} K\right)$ is a subspace for $j=1$ and 2. Consider the case $j=2$, and suppose $(v, y) \in \mathrm{ri}\left(\operatorname{dom}_{2} M\right)$. Then the function $(w, u, x) \rightarrow-M(u, x, v, y)$ is closed proper convex, and by Theorem 8.5 of [10] its recession function is never $-\infty$ and is given by $(w, u, x) \rightarrow$ $-(\operatorname{rec} M(\cdot, \cdot, v, y))(u, x)$. Also, the function $(w, u, x) \rightarrow\langle u, z\rangle+\langle v, w\rangle$ is closed proper convex and coincides with its recession function. Hence Theorem 9.3 of [10] implies that the recession function of $K(z, v, y, \cdot, \cdot, \cdot)$ is given by

$$
(w, u, x) \rightarrow\langle u, z\rangle+\langle v, w\rangle-(\operatorname{rec} M(\cdot, \cdot, v, y))(u, x)
$$

whenever $(v, y) \in \operatorname{ri}\left(\operatorname{dom}_{2} M\right)$, i.e. whenever $(z, v, y) \in \operatorname{ri}\left(\operatorname{dom}_{1} K\right)$. It follows that $(w, u, x) \in A_{2}^{-1}\{0\} \cap$ (rec cone $\left.{ }_{2} K\right)$ if and only if $w=0, x=0$ and $\langle u, z\rangle \leqslant(\operatorname{rec} M(\cdot, \cdot, v, y))(u, 0), \forall z \in Z, \forall(v, y) \in \operatorname{ri}\left(\operatorname{dom}_{2} M\right)$. But since $\operatorname{rec} M(\cdot, \cdot, v, y)$ is never $+\infty$ when $(v, y) \in \operatorname{ri}\left(\operatorname{dom}_{2} M\right)$, in this last condition we must also have $u=0$. Therefore $A_{2}^{-1}\{0\} \cap\left(\operatorname{rec~cone}_{2} K\right)$ is actually the nullspace. By similar reasoning, $A_{1}^{-1}\{0\} \cap\left(\operatorname{rec~cone}_{1} K\right)$ is also the nullspace. The first half of the theorem now follows by taking for $[H]$ the equivalence class $[A K]$ given by Theorem 5 of [4].

To prove the second half of Theorem 1, observe first of all that by the part of the theorem already proved, the functions $M_{1}$ and $M_{2}$ defined in the theorem (for every $H$ in $[H]$ ) belong to a single equivalence class $[N]$ of closed proper concave-convex functions on $(U \times X) \times(V \times Y)$. To complete the proof it suffices to show that $[N]=[M]$. This we shall do in several steps. First, we shall show that $[N]=[B L]$ for appropriate $B$ and $L$. Using this fact, we select a certain element $\widetilde{N}$ of $[N]$. The element $\widetilde{N}$ is shown to satisfy $\underline{M} \leqslant \widetilde{N} \leqslant \bar{M}$, which implies that $\widetilde{N}$ belongs to $[M]$. From this and the definition of equivalence class, it will follow that $[N]=[M]$. We proceed now with the details.

Define a product linear transformation $B=B_{1} \times B_{2}$ and a saddle function $L$ by

$$
B_{1}(u, w, u, x)=(u, x), \quad B_{2}(v, z, v, y)=(v, y),
$$

and

$$
L(\bar{u}, w, u, x, \bar{v}, z, v, y)=\langle z, u-\bar{u}\rangle+\langle w, v-\bar{v}\rangle+\widetilde{M}(\bar{u}, x, \bar{v}, y),
$$

where $\widetilde{M}$ is any element of $[M]$. Clearly $L$ is closed proper concave-convex on $(U \times W \times U \times X) \times(V \times Z \times V \times Y)$. Using the same technique as above, one can easily show that for $j=1$ and 2 the sets $B_{j}^{-1}\{0\} \cap\left(\right.$ rec cone $\left._{j} L\right)$ are 
nullspaces. Hence by Lemma 2 the hypothesis of Theorem 5 of [4] is again satisfied. Let us show that the equivalence class $[B L]$ given by that theorem is exactly $[N]$. For this it suffices to show $[B L]$ contains some element of $[M]$. Now surely $[M]$ contains the function $N$ given by

$$
\begin{aligned}
N(u, x, v, y)= & \sup _{w} \inf _{z}\{\langle z, u\rangle+\langle w, v\rangle \\
& \left.\left.\quad-\inf _{\bar{u}} \sup _{\bar{v}}\{\bar{u}, z\rangle+\langle\bar{v}, w\rangle-\widetilde{M}(\bar{u}, x, \bar{v}, y)\right\}\right\} \\
= & \sup _{w} \inf _{z} \sup _{\bar{u}} \inf _{\bar{v}}\{\langle z, u-\bar{u}\rangle+\langle w, v-\bar{v}\rangle+\widetilde{M}(\bar{u}, x, \bar{v}, y)\} \\
= & \sup _{w} \inf _{z} \sup _{\bar{u}} \inf _{\bar{v}}\{L(\bar{u}, w, u, x, \bar{v}, z, v, y)\} .
\end{aligned}
$$

But by Lemma 36.1 of [10], it is easy to see that $N$ satisfies the inequality

$$
\sup _{B_{1}^{-1}(u, x)} \inf _{B_{2}^{-1}(v, y)} L \leqslant N(u, x, v, y) \leqslant \inf _{B_{2}^{-1}(v, y)} \sup _{B_{1}^{-1}(u, x)} L .
$$

Since the two functions bounding $N$ in this inequality are each in $[B L]$, so is $N$, and hence $[M]=[B L]$. Now observe that the preceding inequality is also valid with $N$ replaced by $\widetilde{N}$, where $\widetilde{N}$ is the following function (obtained from $N$ by commuting some "sup" and "inf" operations):

$$
\widetilde{N}(u, x, v, y)=\sup _{\bar{u}} \inf _{\bar{v}} \sup _{w} \inf _{z}\{L(u, w, u, x, \bar{v}, z, v, y)\} .
$$

Since $\widetilde{N}$ is concave-convex (as can be shown by repeated applications of Theorems 5.5 and 5.7 of $[10]$ ), it follows that $\tilde{N}$ also belongs to $[B L]$, and hence to $[N]$.

Finally, to complete the proof it suffices to show that $\underline{M} \leqslant \tilde{N} \leqslant \bar{M}$. Since the entire argument of the preceding paragraph holds with an arbitrary element $\tilde{M}$ of $[M]$ in the definitions of $L$ and $N$, we can assume that actually $\widetilde{M}=\underline{M}$. Thus, we have that $[N]$ contains the function $\tilde{N}$ given by

$$
\widetilde{N}(u, x, v, y)=\sup _{\bar{u}} \inf _{\bar{v}} \sup _{w} \inf _{z}\{\langle z, u-\bar{u}\rangle+\langle w, v-\bar{v}\rangle+\underline{M}(u, x, \bar{v}, y)\} .
$$

Suppose $u, x, v$ and $y$ are arbitrary but fixed, and define an auxiliary function $p$ by

$$
p(u)=\inf _{\bar{v}} \sup _{w} \inf _{z}\{\langle z, u-\bar{u}\rangle+\langle w, v-\bar{v}\rangle+\underline{M}(\bar{u}, x, \bar{v}, y)\}, \forall \bar{u} \in U .
$$

Observe that

$$
\tilde{N}(u, x, v, y)=\sup \{p(u) \mid \bar{u} \in U(x)\},
$$


where $U(x)=\left\{\bar{u} \in U \mid(\bar{u}, x) \in \operatorname{dom}_{1} M\right\}$. Indeed, if $(\bar{u}, x) \notin \operatorname{dom}_{1} M$, then $\underline{M}(u, x, \cdot, \cdot) \equiv-\infty$ so that $p(u)=-\infty$. Thus,

$$
\widetilde{N}(u, x, v, y)=-\infty=\underline{M}(u, x, v, y)
$$

whenever $U(x)=\varnothing$. Now assume $U(x) \neq \varnothing$. For each $\bar{u} \in U(x)$, $\underline{M}(u, x, \cdot, \cdot)$ is never $-\infty$ and

$$
p(u)=\inf _{\bar{v} \in V(\bar{u})} \sup _{w} \inf _{z}\{\langle z, u-\bar{u}\rangle+\langle w, v-\bar{v}\rangle+\underline{M}(\bar{u}, x, \bar{v}, y)\},
$$

where $V(\bar{u})=\{\bar{v} \in V \mid \underline{M}(\bar{u}, x, \bar{v}, y)<+\infty\}$. This implies that $p(\tilde{u})=+\infty$ whenever $V(u)=\varnothing$. Hence (1) implies that $\widetilde{N}(u, x, v, y)=+\infty$ if there exists a $\bar{u} \in U(x)$ such that $V(u)=\varnothing$. But for such a $\bar{u}, \underline{M}(\bar{u}, x, v, y)=+\infty$ and hence $(v, y) \notin \operatorname{dom}_{2} M$. Therefore

$$
\widetilde{N}(u, x, v, y)=+\infty=\bar{M}(u, x, v, y)
$$

whenever there exists a $\bar{u} \in U(x)$ such that $V(\bar{u})=\varnothing$. Finally, assume $U(x) \neq$ $\varnothing$ and that $V(u) \neq \varnothing$ for every $\bar{u} \in U(x)$. Then for each $\bar{u} \in U(x)$,

$$
p(\bar{u})=\inf _{\bar{v} \in V(\bar{u})}\left\{\underline{M}(\bar{u}, x, \bar{v}, y)+\sup _{w}\left\{\langle w, v-\bar{v}\rangle+\inf _{z}\{\langle z, u-\bar{u}\rangle\}\right\}\right\}
$$

Hence $p(\bar{u})=-\infty$ whenever $u \neq \bar{u} \in U(x)$, while for $u=\bar{u} \in U(x)$ we have that

$$
\begin{aligned}
p(u) & \left.=\inf _{\bar{v} \in V(u)}\left\{\underline{M}(u, x, \bar{v}, y)+\sup _{w}\langle w, v-\bar{v}\rangle\right\}\right\} \\
& =\inf \{\underline{M}(u, x, \bar{v}, y) \mid \bar{v} \in V(u) \text { and } \bar{v}=v\} \\
& = \begin{cases}+\infty & \text { if } v \notin V(u), \\
M(u, x, v, y) & \text { if } v \in V(u) .\end{cases}
\end{aligned}
$$

Hence (1) implies that in this case

$$
\begin{aligned}
\tilde{N}(u, x, v, y) & =\sup \{p(u) \mid \bar{u} \in U(x) \text { and } \bar{u}=u\} \\
& = \begin{cases}\underline{M}(u, x, v, y) & \text { if } u \in U(x) \text { and } v \in V(u), \\
+\infty & \text { if } u \in U(x) \text { and } v \notin V(u), \\
-\infty & \text { if } u \notin U(x),\end{cases} \\
& =\underline{M}(u, x, v, y) .
\end{aligned}
$$

Combining this result with (2) and (3) yields

$$
\underline{M}(u, x, v, y) \leqslant \widetilde{N}(u, x, v, y) \leqslant \bar{M}(u, x, v, y)
$$

in all three cases. Since $u, x, v$, and $y$ were arbitrary, $\underline{M} \leqslant \widetilde{N} \leqslant \bar{M}$ is established and Theorem 1 is proved. 
Observe that in the above proof, the reason for establishing $[N]=[B L]$ was that it essentially allowed us to commute certain "sup" and "inf" operations for an element of $[N]$ and have the resulting function $\widetilde{N}$ remain in $[N]$.

The proofs of the other theorems are shorter than that of Theorem 1. In them we make use of the representation $[H]=[A K]$ obtained in proving Theorem 1.

Proof of Theorem 2. From the proof of Theorem 1 we know that $[H]=$ $[A K]$. It therefore follows from Theorem 6 of [4] that the least element $\underline{H}$ of $[H]$ is given by the formula

$$
\underline{H}(z, y, w, x)=\sup _{v} \mathrm{cl}\left(A_{2} \widetilde{K}(z, v, y, \cdot, \cdot, \cdot)\right)(w, x),
$$

where $\widetilde{K}$ is any element of $[K]$. We can surely confine our attention to those $\widetilde{K}$ expressible as

$$
\widetilde{K}(z, v, y, w, u, x)=\langle u, z\rangle+\langle v, w\rangle-\widetilde{M}(u, x, v, y),
$$

where $\tilde{M}$ is an arbitrary element of $[M]$. Suppose $z, v$ and $y$ are fixed, and define

$$
f(w, x)=\left(A_{2} \widetilde{K}(z, v, y, \cdot, \cdot \cdot \cdot)\right)(w, x) .
$$

Then $f(w, x)=f_{1}(w)+f_{2}(x)$, where

$$
f_{1}(w)=\langle v, w\rangle, \quad f_{2}(x)=\inf _{u}\{\langle u, z\rangle-\widetilde{M}(u, x, v, y)\} .
$$

Moreover, by considering the three cases (i) $f_{2} \equiv+\infty$, (ii) $f_{2}$ somewhere assumes the value $-\infty$, and (iii) $f_{2}$ is proper, it is easy to see that $(\mathrm{cl} f)(w, x)=$ $f_{1}(w)+\left(\mathrm{cl} f_{2}\right)(x)$. Using the notation introduced preceding Theorem 2, we can conclude that

$$
(\mathrm{cl} f)(w, x)=\mathrm{cl}_{x} \inf _{u}\{\langle u, z\rangle+\langle v, w\rangle-\tilde{M}(u, x, v, y)\} .
$$

Combining this formula with the above formula for $\underline{H}$, we obtain that

$$
\underline{H}(z, y, w, x)=\sup _{v} \mathrm{cl}_{x} \inf _{u}\{\langle u, z\rangle+\langle v, w\rangle-\tilde{M}(u, x, v, y)\}
$$

for any element $\widetilde{M}$ of $[M]$. The proof of the formula for $\bar{H}$ is analogous.

Proof of ThEOREM 3. From the proof of Theorem 1 we already have that $[H]=[A K]$. Moreover, Theorem 5 of $[4]$ also gives the conjugacy formula $\left[H^{*}\right]=\left[K^{*} A^{*}\right]$, while Theorem 4 of [4] gives the subdifferential formula $\partial\left(K^{*} A^{*}\right)(\cdot, \cdot)=A \partial K^{*}\left(A^{*}(\cdot, \cdot)\right)$. Since $\partial H^{*}$ is the inverse of $\partial H$ in the sense of multivalued mappings (Theorem 37.5 of [10]), it follows that $(u,-t, v,-s) \in$ $\partial H(z, y, w, x)$ occurs if and only if $(z, y, w, x) \in \partial H^{*}(u,-t, v,-s)$, which occurs if and only if $(z, y, w, x)=A(z, \hat{v}, y, w, \hat{u}, x)$ for some $\hat{u}$ and $\hat{v}$ 
satisfying $(z, \hat{v}, y, w, \hat{u}, x) \in \partial K^{*}\left(A^{*}(u,-t, v,-s)\right)$ that is, if and only if there exist $\hat{u}$ and $\hat{v}$ such that $(u, 0,-t, v, 0,-s) \in \partial K(z, \hat{v}, y, w, \hat{u}, x)$. Now by Theorem 37.4 of [10], this latter containment is equivalent to the condition that $(K(z, \hat{v}, y, w, \hat{u}, x) \in R$ and) the saddle function

$$
K-\langle\cdot,(u, 0,-t)\rangle-\langle\cdot,(v, 0,-s)\rangle
$$

have $(z, \hat{v}, y, w, \hat{u}, x)$ as a saddle point. By the definition of $K$, it follows that $(u,-t, v,-s) \in \partial H(z, y, w, x)$ occurs if and only if there exist $\hat{u}$ and $\hat{v}$ such that $(M(\hat{u}, x, \hat{v}, y) \in R$ and $)$

$$
\begin{aligned}
& M(u, \bar{x}, \hat{v}, y)-\langle\bar{u}-u, z\rangle-\langle\hat{v}-v, \bar{w}\rangle-\langle\bar{x}-x, s\rangle \\
& \quad \leqslant M(\hat{u}, x, \hat{v}, y)-\langle\hat{u}-u, z\rangle-\langle\hat{v}-v, w\rangle \\
& \quad \leqslant M(\hat{u}, x, \bar{v}, \bar{y})-\langle\hat{u}-u, \bar{z}\rangle-\langle\bar{v}-v, w\rangle-\langle y-y, t\rangle
\end{aligned}
$$

for all $(\bar{z}, \bar{v}, \bar{y})$ and all $(\bar{w}, \bar{u}, \bar{x})$. Now pick any $\left(v^{\prime}, y^{\prime}\right) \in \operatorname{dom}_{2} M$. Choosing $\bar{v}=$ $v^{\prime}$ and $\bar{y}=y^{\prime}$ in the above condition implies that

$$
M\left(\hat{u}, x, v^{\prime}, y^{\prime}\right) \geqslant \alpha+\langle\hat{u}-u, \bar{z}\rangle, \quad \forall \bar{z} \in Z,
$$

where $\alpha$ is a certain real constant. If $\hat{u}$ were different from $u$, this would force $. M\left(\hat{u}, x, v^{\prime}, y^{\prime}\right)=+\infty$, contradicting $\left(v^{\prime}, y^{\prime}\right) \in \operatorname{dom}_{2} M$. Therefore in the above condition we must actually have $\hat{u}=u$, and similarly $\hat{v}=v$. Hence, the condition $(u,-t, v,-s) \in \partial H(z, y, w, x)$ is equivalent to the two inequality conditions

$$
M(u, \bar{x}, v, y)-\langle\bar{u}-u, z\rangle-\bar{x}-x, s\rangle \leqslant M(u, x, v, y), \quad \forall(\bar{u}, \bar{x}),
$$

and

$$
M(u, x, v, y) \leqslant M(u, x, \bar{v}, \bar{y})-\langle\bar{v}-v, w\rangle-\langle\bar{y}-y, t\rangle, \quad \forall(\bar{v}, \bar{y}) .
$$

But by Theorem 37.4 of [10] once more, these last conditions are equivalent to $(z, s, w, t) \in \partial M(u, x, v, y)$. This establishes the first assertion of the theorem. Now observe that by choosing $\bar{x}=x$ and $\bar{y}=y$ in the last two inequality conditions, we can deduce that

$$
\begin{aligned}
\langle u, z\rangle+\langle\bar{v}, w\rangle-M(u, x, \bar{v}, y) & \leqslant\langle u, z\rangle+\langle v, w\rangle-M(u, x, v, y) \\
& \leqslant\langle u, z\rangle+\langle v, w\rangle-M(u, x, v, y)
\end{aligned}
$$

for all $\bar{u}$ and all $\bar{v}$, that is, the pair $(u, v)$ forms a saddle point of the saddle function $(\bar{u}, \bar{v}) \rightarrow\langle\bar{u}, z\rangle+\langle\bar{v}, w\rangle-M(\bar{u}, x, \bar{v}, y)$. Furthermore, from the definitions of $H_{1}$ and $H_{2}$, it follows that the saddle value in this case is precisely equal to $H_{j}(z, y, w, x)$ for $j=1$ and 2 . This establishes assertions (i) and (iii) for certain elements of $[M]$ and $[H]$. The refinements to arbitrary elements of these equivalence classes follows easily by Corollary 37.4 .1 of [10]. Thus, only assertion (ii) remains. But this follows from the part of the theorem already proved 
by interchanging the roles of $[M]$ and $[H]$ : because (by Theorem 1 ) the equivalence class $[M]$ is the partial conjugate of $[H]$ in $Z$ and $W$.

Proof of Theorem 4. From the proof of Theorem 1, we have $[H]=$ [AK], and from Theorem 5 of [4] it follows that $\operatorname{dom} H \subset A$ dom $K$. Thus,

$$
\operatorname{dom}_{1} H \subset A_{1} \operatorname{dom}_{1} K=\left\{(z, y) \mid(v, y) \in \operatorname{dom}_{2} M \text { for some } v\right\}
$$

and

$$
\operatorname{dom}_{2} H \subset A_{2} \operatorname{dom}_{2} K=\left\{(w, x) \mid(u, x) \in \operatorname{dom}_{1} M \text { for some } u\right\} \text {. }
$$

These inclusions clearly imply

$$
\operatorname{proj}_{Y}\left(\operatorname{dom}_{1} H\right) \subset \operatorname{proj}_{Y}\left(\operatorname{dom}_{2} M\right) \text { and } \operatorname{proj}_{X}\left(\operatorname{dom}_{2} H\right) \subset \operatorname{proj}_{X}\left(\operatorname{dom}_{1} M\right) \text {. }
$$

On the other hand, since the partial conjugacy correspondence is involutory (Theorem 1), we can interchange the roles of $[H]$ and $[M]$ in the result just established. This yields

$\operatorname{proj}_{X}\left(\operatorname{dom}_{1} M\right) \subset \operatorname{proj}_{X}\left(\operatorname{dom}_{2} H\right)$ and $\operatorname{proj}_{Y}\left(\operatorname{dom}_{2} M\right) \subset \operatorname{proj}_{Y}\left(\operatorname{dom}_{1} H\right)$.

Together with the preceding inclusions, these establish the theorem.

For the proof of Theorem 5 we shall need several lemmas.

LEMMA 3. The closure of any convex function $f$ satisfies

Similarly,

$$
(\mathrm{cl} f)(u)=\sup _{z} \inf _{\bar{u}}\{\langle\bar{u}-u, z\rangle+f(\bar{u})\}
$$

$$
(\operatorname{cl} g)(v)=\inf _{w} \sup _{\bar{v}}\{\langle\bar{v}-v, w\rangle+g(v)\}
$$

for any concave function $g$.

Proof. This is immediate from the basic facts $\mathrm{cl} f=\left(f^{*}\right)^{*}$ and $\mathrm{cl} g=$ $\left(g^{*}\right)^{*}$, together with the definitions of conjugacy for convex and concave functions.

LEMMA 4. The convex and concave parents of a closed saddle function $K$ and its conjugate $K^{*}$ satisfy the identities $f_{K^{*}}=-g_{K}$ and $g_{K^{*}}=-f_{K^{\prime}}$.

PROof. This follows from Theorem 37.1 of [10] and the definitions of convex and concave parent.

Lemma 5. Let $K$ and $A$ be as in Lemma 2, and assume (range $A^{*}$ ) $\cap$ ri (dom $\left.K^{*}\right) \neq \varnothing$. Let $f$ and $h$ (resp. $g$ and $k$ ) denote the convex (resp. concave) parents of $K^{*}$ and $K^{*} A^{*}$. Then $h(z, v)=\left(A_{2} f\left(A_{1}^{*} z, \cdot\right)\right)(v)$ and $k(u, w)=\left(A_{1} g\left(\cdot, A_{2}^{*} w\right)\right)(u)$.

Proof. By Theorem 4 of [4], we know that $\operatorname{dom} K^{*} A^{*}=A^{*-1} \operatorname{dom} K^{*}$. Suppose $z \notin \operatorname{dom}_{1} K^{*} A^{*}$. Then $h(z, \cdot)$ is constantly $+\infty$. Also, $A_{1}^{*} z \notin$ 
$\operatorname{dom}_{1} K^{*}$ implies $f\left(A_{1}^{*} z, \cdot\right)$ is constantly $+\infty$ and hence

$$
\left.\left(A_{2} f\left(A_{1}^{*} z, \cdot\right)\right)(v)=\inf f\left(A_{1}^{*} z, y\right) \mid A_{2} y=v\right\}=+\infty, \quad \forall v .
$$

Now suppose $z \in \operatorname{dom}_{1} K^{*} A^{*}$. Since $A_{1}^{*} z \in \operatorname{dom}_{1} K^{*}$, Theorem 34.3 of [10] implies that $K^{*}\left(A_{1}^{*} z, \cdot\right)$ is a proper convex function with $\operatorname{ri}\left(\operatorname{dom} K^{*}\left(A_{1}^{*} z, \cdot\right)\right)=$ ri $\left(\operatorname{dom}_{2} K^{*}\right)$. Hence, from the hypothesis range $A_{2} \cap \operatorname{ri}\left(\operatorname{dom}_{2} K^{*}\right) \neq \varnothing$ and Theorem 16.3 of [10] it follows that

$$
h(z, v)=\left(K^{*}\left(A_{1}^{*} z, \cdot\right) A_{2}\right)^{*}(v)=\left(A_{2} K^{*}\left(A_{1}^{*} z, \cdot\right)^{*}\right)(v)=\left(A_{2} f\left(A_{1}^{*} z, \cdot\right)\right)(v)
$$

for every $v$. This establishes the first identity. The second is proved similarly.

Proof of THEOREM 5. Suppose that we have already shown that $-g_{H}(u, t, w, x) \geqslant f_{M}(u, x, w,-t)$ and $-f_{H}(z, y, v, s) \leqslant g_{M}(z,-s, v, y)$. Since the partial conjugacy correspondence is involutory (by Theorem 1), we can interchange the roles of $[H]$ and $[M]$ in these inequalities to deduce

$$
-g_{M}(z, s, v, y) \geqslant f_{H}(z, y, v,-s) \text { and }-f_{M}(u, x, w, t) \leqslant g_{H}(u,-t, w, x) \text {. }
$$

From these four inequalities the two identities in the theorem are immediate. To complete the proof, it therefore suffices to establish the first two inequalities above. We shall demonstrate only the first, as the proof of the second is entirely analogous. From Lemma 5 we have the identity

$$
f_{H^{*}}(u, t, w, x)=\left(A_{2} f_{K^{*}}\left(A_{1}^{*}(u, t), \cdot\right)\right)(w, x) .
$$

Since $f_{H^{*}}=-g_{H}$ and $f_{K^{*}}=-g_{K}$ by Lemma 4 , this identity can be rewritten as

$$
-g_{H}(u, t, w, x)=\inf _{\bar{u}}\left\{\left(-g_{K}\right)(u, 0, t, w, \bar{u}, x)\right\} .
$$

Now by the definition of $K, g_{K}(u, 0, t, w, \bar{u}, x)$ is equal to

$$
\inf _{z, v, y}\{\langle z, u\rangle+\langle v, 0\rangle+\langle y, t\rangle-(\langle u, z\rangle+\langle v, w\rangle-M(\tilde{u}, x, v, y))\}
$$

which equals

that is,

$$
\inf _{z}\left\{\langle u-\bar{u}, z\rangle+\inf _{v, y}\{-\langle v, w\rangle+\langle y, t\rangle+M(u, x, v, y)\}\right\},
$$

$$
\inf _{z}\left\{\langle u-\bar{u}, z\rangle-\sup _{v, y}\{\langle v, w\rangle+\langle y,-t\rangle-M(\bar{u}, x, v, y)\}\right\} .
$$

From the definition of convex parent it follows that

$$
g_{K}(u, 0, t, w, \bar{u}, x)=\inf _{z}\left\{\langle u-\bar{u}, z\rangle-f_{M}(\bar{u}, x, w,-t)\right\} .
$$


Therefore

$$
\begin{aligned}
-g_{H}(u, t, w, x) & =\inf _{\bar{u}} \sup _{z}\left\{\langle\bar{u}-u, z\rangle+f_{M}(\bar{u}, x, w,-t)\right\} \\
& \geqslant \sup _{z} \inf _{\bar{u}}\left\{\langle\bar{u}-u, z\rangle+f_{M}(\bar{u}, x, w,-t)\right\},
\end{aligned}
$$

where the inequality is by Lemma 36.1 of [10]. Finally, consider the function $f$ given by $f=f_{M}(\cdot, x, w,-t)$. Since $f_{M}$ is closed and convex, so is $f$. Hence, applying Lemma 3 with this $f$, we obtain

$$
-g_{H}(u, t, w, x) \geqslant(\operatorname{cl} f)(u)=f(u)=f_{M}(u, x, w,-t),
$$

as desired.

\section{REFERENCES}

1. A. Brondsted, Conjugate convex functions in topological vector spaces, Mat.-Fys. Medd. Danske Vid. Selsk. 34 (1964), no. 2, 27pp. MR 29 \#3853.

2. W. Fenchel, On conjugate convex functions, Canad. J. Math. 1 (1949), 73-77. MR 10, 435.

3. Convex cones, sets and functions, mimeographed lecture notes, Princeton University, 1951.

4. L. McLinden, Dual operations on saddle functions, Trans. Amer. Math. Soc. 179 (1973), 363-381. MR 47 \#4645.

5. - Minimax problems, saddle functions and duality, Thesis, University of Washington, 1971; revised and expanded as Technical Summary Report \#1190, Math. Res. Center, University of Wisconsin, Madison, Wis., 1971.

6. J.-J. Moreau, Théorèmes "inf-sup", C. R. Acad. Sci. Paris 258 (1964), 2720-2722. MR 28 \#4336.

7. - Fonctionelles convexes, lecture notes, Séminaire sur "Lés équations aux dérivées partielles," Collège de France, 1966.

8. R. T. Rockafellar, Minimax theorems and conjugate saddle-functions, Math. Scand. 14 (1964), 151-173. MR 30 \#5223; erratum, 31, p. 1336.

9. - A general correspondence between dual minimax problems and convex programs, Pacific J. Math. 25 (1968), 597-611. MR 37 \#6110.

10. Convex analysis, Princeton Math. Ser., no. 28, Princeton Univ. Press, Princeton, N. J., 1970. MR 43 \#445.

11. - Saddle-points and convex analysis, Differential Games and Related Topics (Proc. Internat. Summer School, Varenna, 1970), North-Holland, Amsterdam, 1971, pp. 109-127. MR 44 \#3164.

MATHEMATICS RESEARCH CENTER, UNIVERSITY OF WISCONSIN, MADISON, WISCONSIN 53706

Current address: Department of Mathematics, University of Illinois at Urbana-Champaign, Urbana, Illinois 61801 